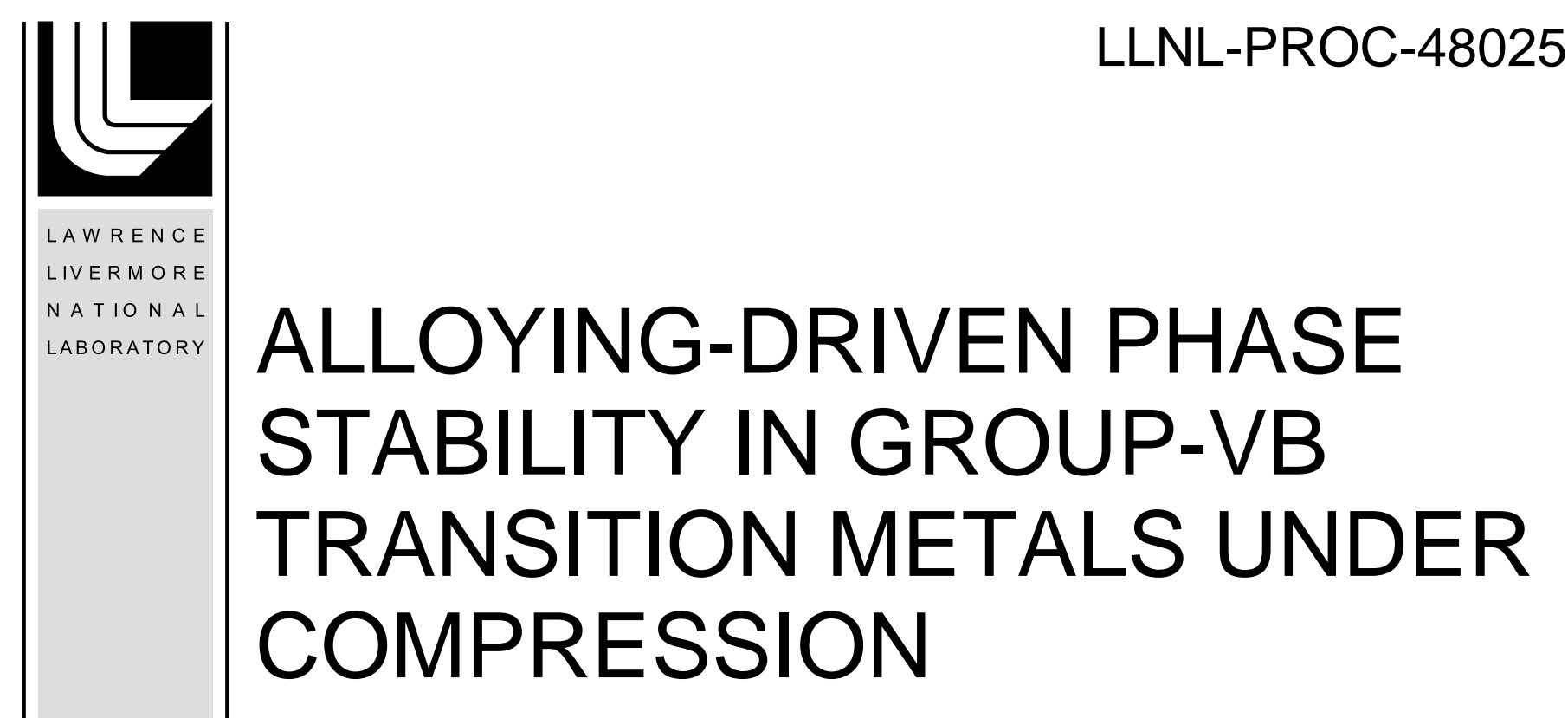

A. Landa, P. Soderlind

April 13, 2011

2011 MRS Spring Meeting

San Francisco, CA, United States

April 25, 2011 through April 29, 2011 
This document was prepared as an account of work sponsored by an agency of the United States government. Neither the United States government nor Lawrence Livermore National Security, LLC, nor any of their employees makes any warranty, expressed or implied, or assumes any legal liability or responsibility for the accuracy, completeness, or usefulness of any information, apparatus, product, or process disclosed, or represents that its use would not infringe privately owned rights. Reference herein to any specific commercial product, process, or service by trade name, trademark, manufacturer, or otherwise does not necessarily constitute or imply its endorsement, recommendation, or favoring by the United States government or Lawrence Livermore National Security, LLC. The views and opinions of authors expressed herein do not necessarily state or reflect those of the United States government or Lawrence Livermore National Security, LLC, and shall not be used for advertising or product endorsement purposes. 


\title{
Alloying-Driven Phase Stability in Group-VB Transition Metals under Compression
}

\author{
Alexander Landa ${ }^{1}$ and Per Söderlind ${ }^{1}$ \\ ${ }^{1}$ Condensed Matter and Materials Division, Physical and Life Sciences Directorate, Lawrence \\ Livermore National Laboratory, L-045, 7000 East Avenue, Livermore, CA 94551-0808, U.S.A.
}

\begin{abstract}
The change in phase stability of Group- $\mathrm{VB}(\mathrm{V}, \mathrm{Nb}$, and $\mathrm{Ta})$ transition metals due to pressure and alloying is explored by means of first-principles electronic-structure calculations. It is shown that under compression stabilization or destabilization of the ground-state bodycentered cubic (bcc) phase of the metal is mainly dictated by the band-structure energy that correlates well with the position of the Kohn anomaly in the transverse acoustic phonon mode. The predicted position of the Kohn anomaly in $\mathrm{V}, \mathrm{Nb}$, and $\mathrm{Ta}$ is found to be in a good agreement with data from the inelastic $\mathrm{x}$-ray or neutron scattering measurements. In the case of alloying the change in phase stability is defined by the interplay between the band-structure and Madelung energies. We show that band-structure effects determine phase stability when a particular GroupVB metal is alloyed with its nearest neighbors within the same $d$-transition series: the neighbor with less and more $d$ electrons destabilize and stabilize the bcc phase, respectively. When $\mathrm{V}$ is alloyed with neighbors of a higher ( $4 d$ - or $5 d$-) transition series, both electrostatic Madelung and band-structure energies stabilize the body-centered-cubic phase. The opposite effect (destabilization) happens when $\mathrm{Nb}$ or Ta is alloyed with neighbors of the $3 d$-transition series.
\end{abstract}

\section{INTRODUCTION}

Vanadium metal has been the subject of numerous experimental and theoretical studies due to its high superconducting transition temperature. Ishizuka et al. [1] found that for $\mathrm{V}$ the superconducting transition temperature, $T_{c}=5.3 \mathrm{~K}$, increases linearly with pressure reaching $17.2 \mathrm{~K}$ (the highest $T_{c}$ among the elemental metals reported so far) at $1.2 \mathrm{Mbar}$.

In order to explore the response of electron-phonon interaction to high pressures, Suzuki and Otani [2] performed first-principles calculations of the lattice dynamics of $\mathrm{V}$ in the pressure range up to $1.5 \mathrm{Mbar}$. They found that the frequencies of the TA mode [ $\xi 00]$ soften around $\xi=1 / 4$ with increasing pressure and become imaginary (unstable) at pressures higher than $1.3 \mathrm{Mbar}$, indicating a structural phase transition. Later this result was confirmed by other ab-initio calculations [3]. On the experimental side, recently by using inelastic $\mathrm{x}$-ray scattering technique Bosak et al. [4] discovered several anomalies in the phonon dispersion curve for $\mathrm{V}$ along highsymmetry directions. Among these anomalies was an upward bending of the transverse acoustic (TA) mode along the $\Gamma-H$ direction, [ $\xi 00]$, around $\xi=0.24$ - the anomaly that has been predicted theoretically in Refs. [2, 3]. The recent synchrotron x-ray diffraction measurements on V [5] reported a new rhombohedral $(r h)$ phase around 630-690. Later theoretical studies confirm this finding and also suggest vanadium to return to the bcc phase around 2.5-2.8 Mbar [3, 6].

The phonon anomaly in the TA [ $\xi 00]$ phonon branch of $\mathrm{V}$ was attributed to the existence of parallel pieces of the Fermi surface (FS) which causes a strong electronic response at the nesting wave-vector that translates these pieces one to the other [7]. The same authors [7] also 
found that the nesting vector $\boldsymbol{q}_{n}$, spanning two flat pieces of the FS in the $3^{\text {rd }}$ band, already exists at zero pressure and leads to the Kohn anomaly in the TA [ $\xi 00]$ phonon mode for small $q_{n} \approx$ $0.24(2 \pi / a)$, where $a$ is the lattice constant. Since in the long wave-length limit $(q \rightarrow 0)$ the trigonal shear elastic constant, $c_{44}$, is related to the TA [ $\left.\xi_{00}\right]$ phonon frequency, $\omega(q)$, by a simple relation: $\rho \omega^{2}(q) / q^{2} \rightarrow c_{44}$, the Kohn anomaly also softens this elastic constant making it negative in the pressure range 1.80-2.75 Mbar. The full potential linear muffin-tin orbitals [8] and projector augmented waves [9] calculations reveal the lower limit of this pressure interval as 1.2 and 0.8 Mbar, respectively.

The remaining Group-VB transition metals, $\mathrm{Nb}$ and Ta, also represent the so-called hard superconductors whose superconducting properties highly depend on their physical and chemical state. Niobium has the highest superconducting transition temperature, $T_{c}=9.25 \mathrm{~K}$, among the elemental metals at ambient pressure [10]. According to Matthias et al. [11], tantalum becomes a superconductor at $T_{c} \sim 3.39-4.48 \mathrm{~K}$. By using inelastic neutron scattering, Nakagawa and Woods [12] found a decrease (below the elastic constant line) in TA [ $\xi 00]$ phonon frequencies for $\mathrm{Nb}$ around $\xi=0.20$ (compare with $\xi=0.24$ for V). Woods [13] performed the frequency-wavevector dispersion relation measurements in Ta using the inelastic neutron scattering technique similar that was used in measurements for $\mathrm{Nb}$ [12]. His results showed strong analogies with previously measured $\mathrm{Nb}$ [12], however, a drastic decrease below the elastic constant line for TA [ $\xi 00]$ phonon mode, observed for $\mathrm{Nb}$, was not detected.

The question whether the TA [ $\xi 00]$ phonon anomaly in Group-VB transition metals is indeed electronic in nature and connected with 'nesting areas' of the Fermi surface can be answered by comparing the nesting vectors, $\boldsymbol{q}_{\boldsymbol{n}}=2 \boldsymbol{k}_{F}$, in all Group-VB metals with the positions of their phonon anomalies, either calculated or measured experimentally. Luckily, reliable experimental data exist on phonon dispersions in all three Group-VB metals - V [4], $\mathrm{Nb}$ [12], and Ta [13], and it is time to fill this gap.

Another task of this paper is to study stability of Group-VB transition metals due to alloying with a small amount of a neighboring metal, particularly stabilization or destabilization of the bcc phase relative to a lower symmetry $r h$ phase under compression.

\section{THEORY}

\section{Methodology}

The calculations that we have referred to as exact muffin-tin orbitals (EMTO) are performed using a scalar-relativistic Green function technique based on an improved screened Korringa-Kohn-Rostoker method [14]. For the exchange/correlation approximation, we use the generalized gradient approximation (GGA) [15]. In order to treat compositional disorder the EMTO method is combined with the coherent potential approximation $[16,17]$. Integration over the irreducible wedge of the Brillouin zone is performed using the special $k$-points method [18]. These calculations include the on-site screened Coulomb potential and energy, which take care of the electrostatics in the single-site CPA approximation [19]. The corresponding screening constants have been obtained in the locally self-consistent Green function (LSGF) [20] calculations for a 1024 atoms supercell that models the random equiatomic alloys. For the elemental Group-VB metals, the calculations are also performed using a full-potential linear 
muffin-tin orbital method (FPLMTO) [21] with the same choice (GGA) for the exchange/correlation. The equilibrium density and EOS are obtained from a Murnaghan fit [22] to the total-energy versus the lattice constant curve. For the determination of the trigonal shear elastic constant, $c_{44}$, we apply a volume-conserving monoclinic distortion [23] and calculate the internal energy response. The ground state for the $r$ phase is obtained by applying a volume conserving rhombohedral distortion [6].

The generalized susceptibility of non-interacting electrons $(\chi(q))$ is selected in order to detect the FS nesting. This function is calculated by using the analytic tetrahedron method [24].

\section{Generalized susceptibility calculation}

The generalized susceptibility of non-interacting electron electrons $\chi(q)$ is closely related to the flat patches of the FS separated by some (nesting) vector $2 \boldsymbol{k}_{F}$ peaking precisely at $\boldsymbol{q}=2 \boldsymbol{k}_{F}$. Therefore, if the positions of some phonon anomalies of a metal system are closely correlated with the positions of maximum in $\chi(q)$, this would strongly indicate that these anomalies are of the Kohn nature.

Figure 1 shows the partial (due to the $3^{\text {rd }} \rightarrow 3^{\text {rd }}$ electron intra-band transition, see Ref. [7, 8] for details) contribution to the generalized susceptibility, $\chi(q)$, of $\mathrm{V}, \mathrm{Nb}$, and Ta calculated along $\Gamma-H(\xi 00)$ direction at ambient pressure. As can be seen from this plot, the generalized susceptibility has a peak at $\xi \approx 0.24,0.16$, and 0.28 for $\mathrm{V}, \mathrm{Nb}$, and $\mathrm{Ta}$, respectively, indicating the position where the Kohn anomaly on the TA [ $\xi 00]$ phonon branch is likely to occur. One can also see that this peak is more pronounced for $\mathrm{V}$ and $\mathrm{Nb}$ than for Ta suggesting that the Kohn anomaly is weaker in Ta than in $\mathrm{V}$ and $\mathrm{Nb}$. In order to verify these results, we plot in figure $2 \mathrm{a}$, figure $2 b$, and figure $2 c$ the experimental phonon dispersion TA curve in [ $\xi 00$ ] direction for $V$, $\mathrm{Nb}$, and $\mathrm{Ta}$, respectively, using numerical data on the phonon frequencies from Refs. [13, 25 , and 26]. The location of the peak on the partial generalized susceptibility curve at ambient pressure, presented in figure 1 , is marked by an arrow in figures 2 . One can see that the position of the calculated peak in $\chi(q)$ is in fair accord with the point of the maximum deviation below the elastic constant line of the experimental TA [ $\xi 00]$ phonon branch (the so-called phonon softening). In comparison with $\mathrm{V}$, this deviation is observed to a larger and smaller extent for $\mathrm{Nb}$ and $\mathrm{Ta}$, respectively.

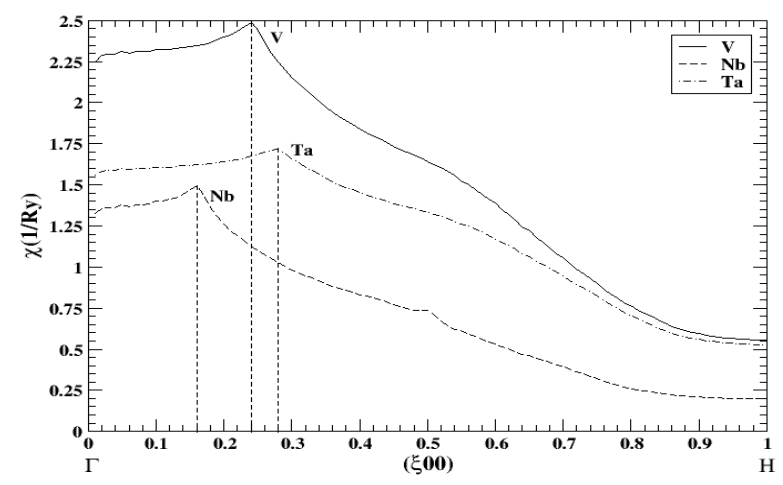

Figure 1. Partial $\left(3^{\text {rd }} \rightarrow 3^{\text {rd }}\right.$ intra-band transition) electron susceptibility of $\mathrm{V}, \mathrm{Nb}$, and $\mathrm{Ta}$ calculated along the $\Gamma-H$ direction at ambient pressure. 

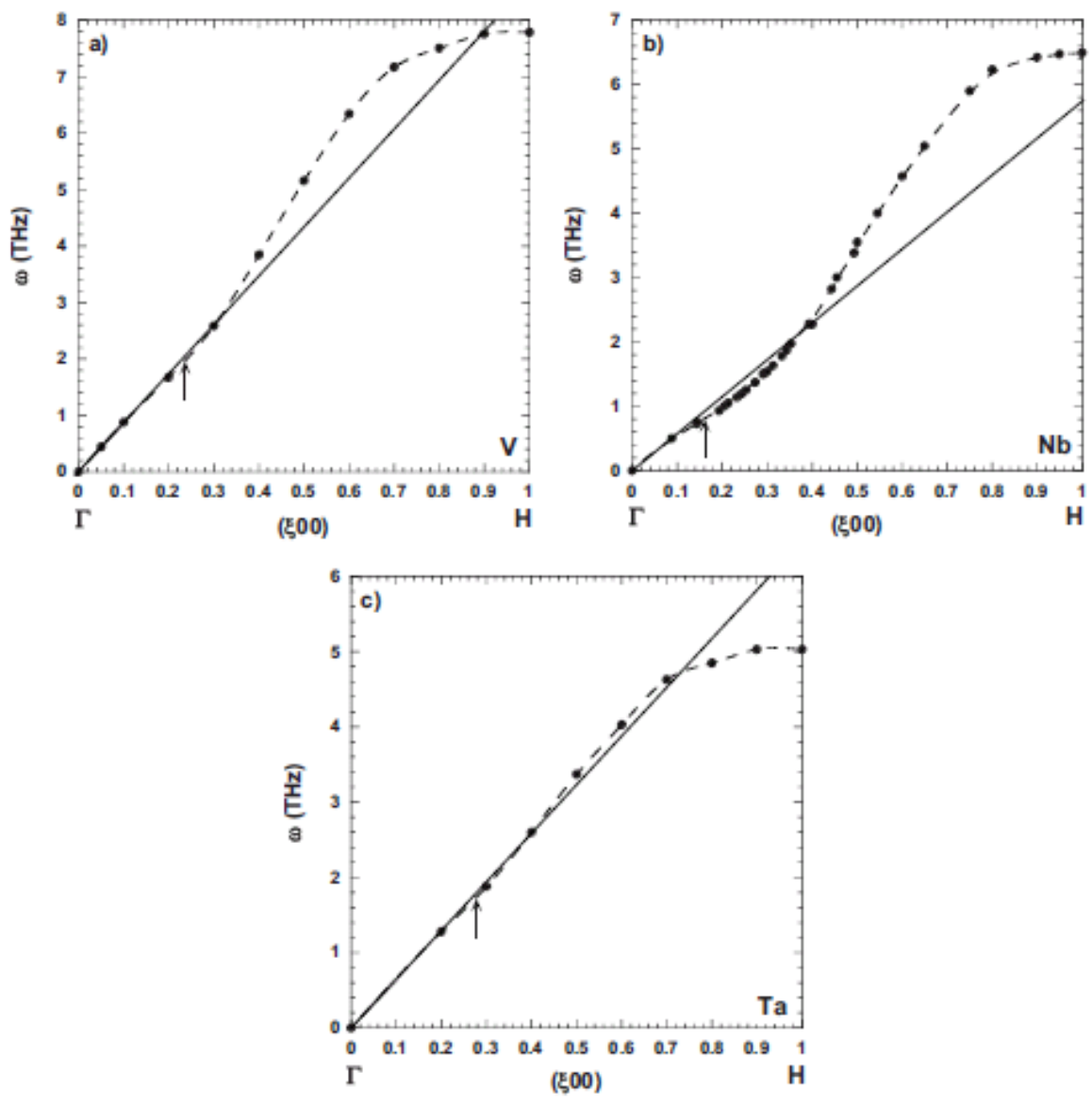

Figure 2. The experimental phonon dispersion TA curve in [ $\xi 00$ ] direction for a) V (Ref. [25]), b) $\mathrm{Nb}$ (Ref. [26]), and c) Ta (Ref. [13]). The arrow indicates the Kohn anomaly obtained from $a b$ initio calculations. The straight line represents the elastic constant line.

\section{Stability of the bcc alloys based on the $\mathrm{V}, \mathrm{Nb}$, and Ta}

Figure 3 shows calculated (FPLMTO) pressure dependence of the trigonal shear elastic constant, $c_{44}$, in $\mathrm{V}, \mathrm{Nb}$, and Ta. For $\mathrm{V}$ we predict the phase transition to the rh phase at $0.6 \mathrm{Mbar}$, close to the observed 0.6-0.7 Mbar [5]. For $\mathrm{Nb}$ the shear constant reaches a low minimum close to $0.5 \mathrm{Mbar}$ before rising again. In Ta the softening is less severe but apparent between 0.5-0.8 Mbar.

In figure 4 we display the EMTO-CPA total energy as a function of rhombohedral distortion for $\mathrm{V}$ and its alloys with 5 at. $\%$ of $\mathrm{Ti}, \mathrm{Cr}$, and $\mathrm{Nb}$. Here the atomic volume is kept at $\Omega$ $=8.056 \AA^{3}$ corresponding to a pressure of $\sim 2.4$ Mbar. According to our calculations, this pressure corresponds to the point of the maximum stability of the $r h$ phase of pure $\mathrm{V}$ in respect with the bcc phase - the ground state phase at ambient pressure. Notice that pure $\mathrm{V}$ is unstable with respect to the distortion and alloying with a small amount of its left neighbor Ti increases the instability. Adding a small fraction of $\mathrm{Cr}$ or $\mathrm{Nb}$, however, promotes the bcc phase which becomes stable for $\sim 5$ at. $\% \mathrm{Nb}$ and $\sim 11$ at. $\% \mathrm{Cr}$ (not shown). Our calculations also predict that $\mathrm{Zr}$ ( 3 at.\%), Mo ( 5 at.\%), Hf ( 5 at.\%), Ta ( 5 at.\%), and W ( 4 at. $\%)$ stabilize the bcc 
phase of $\mathrm{V}$ at all pressures. Thus all the metals in Group-IVB, Group-VB, and Group-VIB prevent the rhombohedral phase in $\mathrm{V}$, except $\mathrm{Ti}$, which in contrast favors the distorted phase.

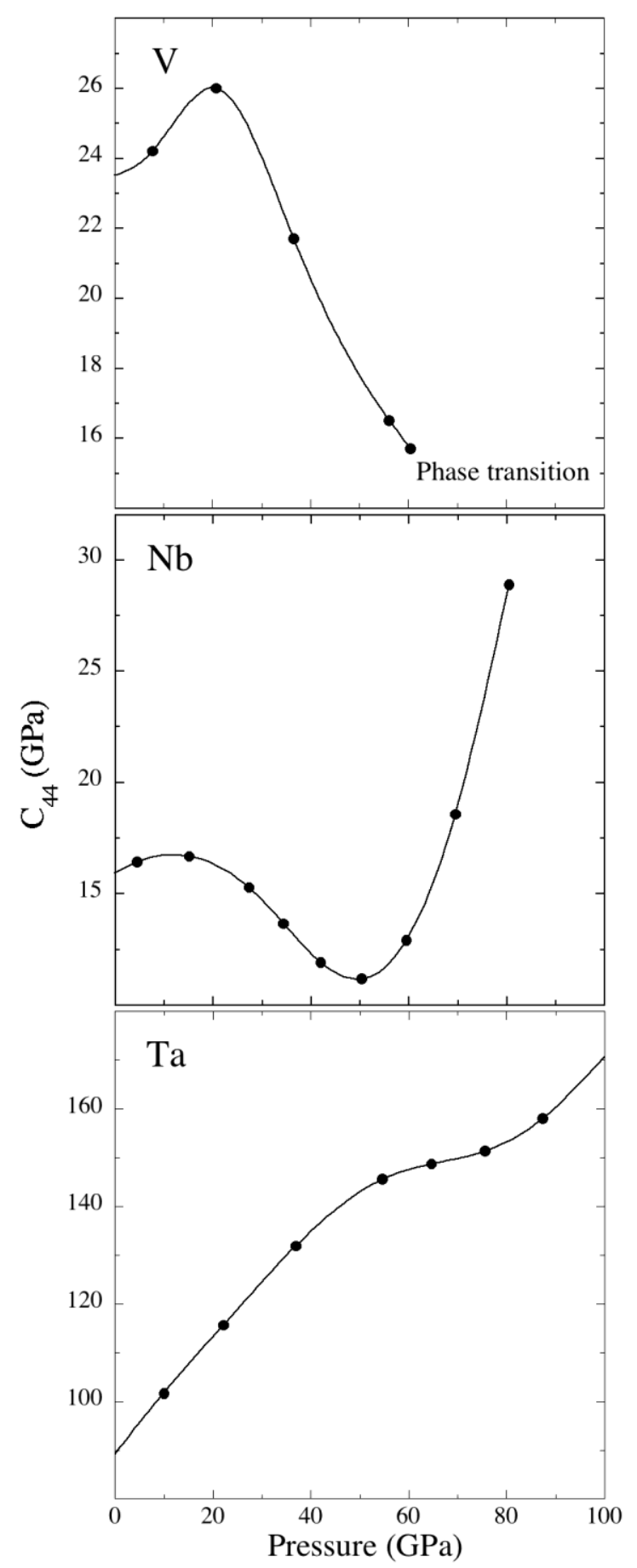

Figure 3. Calculated (FPLMTO) pressure dependence of the shear elastic constant in V, Nb, and Ta. 


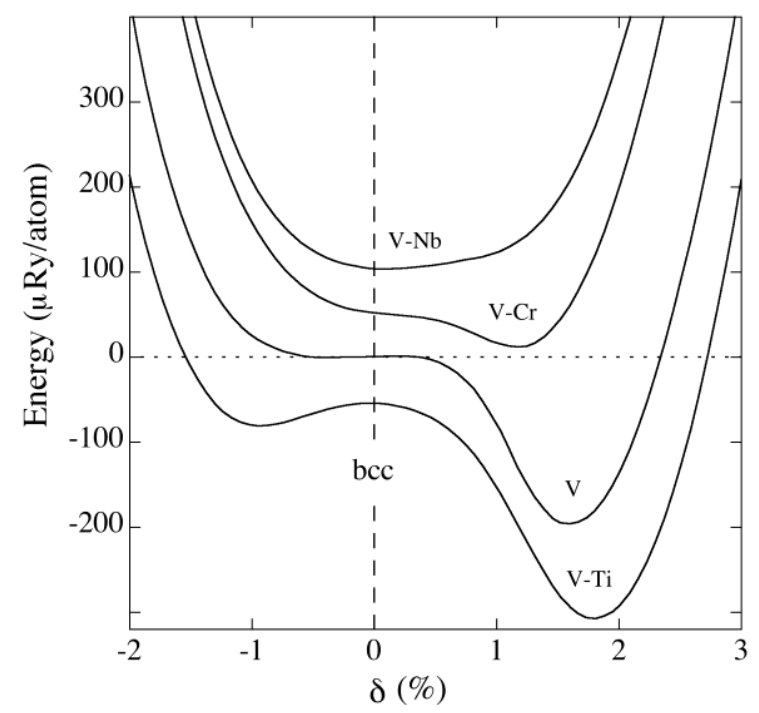

Figure 4. Calculated total energy (at the atomic volume $\Omega=8.056 \AA^{3}$ ) as a function of the rhombohedral deformation parameter $\delta$ (see Ref. [6] for explanation). The undistorted ( $\delta=0)$ crystal corresponds to the bcc phase. The curves for $\mathrm{V}_{95} \mathrm{Ti}_{05}, \mathrm{~V}_{95} \mathrm{Cr}_{05}$, and $\mathrm{V}_{95} \mathrm{Nb}_{05}$ are shifted apart $50 \mathrm{mRy}$ for a better display of the results.

Within the EMTO formalism, the total-energy, $E_{t o t}$, can be expressed as the sum of two contributions: $E_{t o t}=E_{1}+E_{M}$, where $E_{1}$ consists of all "local" (band-structure) contributions, $E_{1}=$ $E_{s}+E_{\text {intra }}+E_{x c}$, such as the kinetic energy of non-interacting electron gas, $E_{s}$, the intra-cell electrostatic energy, $E_{\text {intra }}$, which is due to the electron-electron and electron-ion Coulomb interactions and also includes the screened Coulomb interactions in the case of the DFT-CPA calculations, and the exchange and correlation energy, $E_{x c}$. The remaining contribution, $E_{M}$, is the inter-cell Madelung energy. Note that the change in $E_{M}$ due to alloying is proportional to the magnitude of the charge transfer between the atoms.

Table I lists the total-energy response, $\Delta E_{\text {tot }}$, and its contributions, $\Delta E_{l}$ and $\Delta E_{M}$, to a $1 \%$ monoclinic deformation, used for the trigonal shear elastic constant $c_{44}$ calculations [23], calculated for $\mathrm{V}, \mathrm{Nb}$, and Ta and their alloys with the Group-IVB (Ti, Zr, and Hf), Group-VB (V, Nb, and Ta) and Group-VIB (Cr, Mo, and W) metals. Negative values of the total-energy changes indicate mechanical instability of the bcc phase. The results for pure $\mathrm{V}, \mathrm{Nb}$, and Ta are presented in plain font. In the case of $\mathrm{V}(\mathrm{Nb}, \mathrm{Ta})$-based alloys the choice of the font depends on whether one observes stabilization or destabilization of the bcc phase: in the case of former (stabilization) scenario the results are presented in bold font and in the case of the later (destabilization) scenario the results are presented in italic font.

The calculations for pure vanadium and V-based alloys are performed at the atomic volume $\Omega=8.056 \AA^{3}$ corresponding to the pressure of $\sim 2.4$ Mbar as was mentioned above. Adding Ti to $\mathrm{V}$ slightly decreases $\Delta E_{\text {tot }}$ signaling further bcc destabilization and the change in the band energy, $E_{1}$, plays the dominant role. For the $\mathrm{V}_{95} \mathrm{Cr}_{05}$ alloy, again the band energy is dominant, now having the opposite effect while the Madelung part remains nearly the same. Consequently, $\Delta E_{\text {tot }}$ increases and approaches zero with a near stabilization of the bcc phase. Adding of an additional 6 at. \% of $\mathrm{Cr}$ in fact dictates the bcc phase for all pressures in $\mathrm{V}$. Thus we can conclude that the band-filling argument (the number of valence electrons) is responsible, 
exclusively, for stabilization or destabilization of the bcc phase when $\mathrm{V}$ is alloyed with its neighbors within the same $3 d$-transition series, $\mathrm{Cr}$ or $\mathrm{Ti}$, respectively.

Table I. Contributions to the total-energy change (in mRy) due to a $1 \%$ monoclinic deformation for $\mathrm{V}, \mathrm{Nb}$, and $\mathrm{Ta}$, and their alloys. The results for pure $\mathrm{V}, \mathrm{Nb}$, and $\mathrm{Ta}$ are presented in plain font. In the case of $\mathrm{V}(\mathrm{Nb}, \mathrm{Ta})$-based alloys the choice of the font depends on whether one observe stabilization (bold) or destabilization (italic) of the bcc phase.

\begin{tabular}{|c|l|l|l|l|l|l|l|l|l|l|}
\hline \multicolumn{2}{|c|}{ Metal $\rightarrow$} & \multicolumn{3}{c|}{ Group-IVB } & \multicolumn{3}{c|}{ Group-VB } & \multicolumn{3}{c|}{ Group-VIB } \\
\cline { 3 - 11 } \multicolumn{2}{|c|}{ Alloy $\downarrow$} & Ti $3 d$ & Zr $4 d$ & Hf $5 d$ & V $3 d$ & Nb $4 d$ & Ta $5 d$ & Cr $3 d$ & Mo $4 d$ & W $5 d$ \\
\hline \multirow{3}{*}{$\mathrm{V}_{95} \mathrm{M}_{05}$} & $\Delta E_{l}$ & -.2130 & $\mathbf{- . 1 8 6 4}$ & $\mathbf{- . 1 9 3 1}$ & -.2010 & $\mathbf{- . 1 9 5 1}$ & $\mathbf{- . 1 6 6 2}$ & $\mathbf{- . 1 8 8 7}$ & $\mathbf{- . 1 8 5 5}$ & $\mathbf{- . 1 1 5 0}$ \\
& $\Delta E_{M}$ & $\mathbf{. 1 8 9 5}$ & $\mathbf{. 2 1 2 1}$ & $\mathbf{. 2 0 4 4}$ & .1820 & $\mathbf{. 2 1 0 2}$ & $\mathbf{. 2 0 0 3}$ & $\mathbf{. 1 8 2 3}$ & $\mathbf{. 1 9 3 9}$ & $\mathbf{. 1 9 1 5}$ \\
& $\Delta E_{t o t}$ & -.0235 & $\mathbf{. 0 2 5 7}$ & $\mathbf{. 0 1 1 3}$ & -.0190 & $\mathbf{. 0 1 5 1}$ & $\mathbf{. 0 3 4 1}$ & $\mathbf{- . 0 0 6 4}$ & $\mathbf{. 0 0 8 4}$ & $\mathbf{. 0 7 6 5}$ \\
\hline \multirow{3}{*}{$\mathrm{Nb}_{95} \mathrm{M}_{05}$} & $\Delta E_{l}$ & -.0940 & -.1005 & -.0946 & -.0905 & -.0860 & $\mathbf{- . 0 8 4 0}$ & -.0896 & $\mathbf{- . 0 8 0 8}$ & $\mathbf{- . 0 8 4 6}$ \\
& $\Delta E_{M}$ & .1839 & .1851 & .1861 & .1829 & .1930 & $\mathbf{. 1 9 3 5}$ & .1876 & .1910 & .1850 \\
& $\Delta E_{t o t}$ & .0899 & .0846 & .0915 & .0924 & .1070 & $\mathbf{. 1 0 9 5}$ & .0890 & $\mathbf{. 1 1 0 2}$ & .1004 \\
\hline \multirow{3}{*}{$\mathrm{Ta}_{90} \mathrm{M}_{10}$} & $\Delta E_{l}$ & -.0188 & -.0223 & -.0169 & -.0181 & -.0103 & -.0080 & -.0161 & -.0130 & $\mathbf{. 0 0 0 0}$ \\
& $\Delta E_{M}$ & .2194 & $\mathbf{. 2 2 7 7}$ & $\mathbf{. 2 2 8 9}$ & .2145 & $\mathbf{. 2 3 1 6}$ & .2250 & .2117 & .2179 & .2250 \\
& $\Delta E_{t o t}$ & .2006 & .2054 & .2120 & .1964 & $\mathbf{. 2 2 1 3}$ & .2170 & .1956 & .2049 & $\mathbf{. 2 2 5 0}$ \\
\hline
\end{tabular}

Adding $4 d$-transition metals ( $\mathrm{Zr}$, Nb, or $\mathrm{Mo}$ ) to $\mathrm{V}$ significantly increases $\Delta E_{\text {tot }}$ thus implying stabilization of the bcc structure in the $\mathrm{V}_{95} \mathrm{Zr}_{05}, \mathrm{~V}_{95} \mathrm{Nb}_{05}$, or $\mathrm{V}_{95} \mathrm{Mo}_{05}$ alloys. Notice that the increase of $\Delta E_{1}$ contribution for the $\mathrm{V}_{95} \mathrm{Nb}_{05}$ alloy in comparison with pure $\mathrm{V}$, which is mostly due to band-structure effects, is rather insignificant $(+0.0059 \mathrm{mRy})$ and the main reason for stabilization of the bcc structure in this alloy is instead the increase of the electrostatic Madelung energy contribution, $\Delta E_{M},(+0.0282 \mathrm{mRy})$.

For the $\mathrm{V}_{95} \mathrm{Zr}_{05}$ or $\mathrm{V}_{95} \mathrm{Mo}_{05}$ alloys, the increase in the band energy contribution, $\Delta E_{1}$, is, on average, 2.5 times larger than for the $\mathrm{V}_{95} \mathrm{Nb}_{05}$ alloy where this contribution is rather insignificant. Although the increase in $\Delta E_{M}$, which, as was already mentioned, is proportional to the magnitude of the charge transfer on the vanadium atoms (see table II), is significant: $+0.0119,+0.0282$, and $+0.0301 \mathrm{mRy}$ for the $\mathrm{V}_{95} \mathrm{Mo}_{05}, \mathrm{~V}_{95} \mathrm{Nb}_{05}$, or $\mathrm{V}_{95} \mathrm{Zr}_{05}$ alloys, respectively, the increase in $\Delta E_{1}$ for the $\mathrm{V}_{95} \mathrm{Mo}_{05}$ alloy $(+0.0155 \mathrm{mRy})$ is even larger than that in $\Delta E_{M}$ $(+0.0119 \mathrm{mRy})$. So for the $\mathrm{V}_{95} \mathrm{Zr}_{05}$ or $\mathrm{V}_{95} \mathrm{Mo}_{05}$ alloys we cannot credit the electrostatic Madelung energy as the dominating factor in stabilization of the bcc structure in the V-4d-transition metal alloys as we claim in the case of the $\mathrm{V}_{95} \mathrm{Nb}_{05}$ alloy.

Table II. The calculated charge transfer induced on the V atoms in V-M alloys.

\begin{tabular}{|l|l|l|}
\hline V-Ti (0.077) & V-V (0.000) & V-Cr (-0.070) \\
\hline V-Zr (0.290) & V-Nb (0.247) & V-Mo (0.196) \\
\hline V-Hf (0.306) & V-Ta (0.296) & V-W (0.277) \\
\hline
\end{tabular}

Adding $5 d$-transition metals ( $\mathrm{Hf}, \mathrm{Ta}$, and $\mathrm{W}$ ) also stabilizes the bcc structure in the $\mathrm{V}_{95} \mathrm{Hf}_{05}, \mathrm{~V}_{95} \mathrm{Ta}_{05}$, and $\mathrm{V}_{95} \mathrm{~W}_{05}$ alloys. Although the increase in $\Delta E_{M}(+0.0095,+0.0183$, and $+0.0224 \mathrm{mRy}$, for the $\mathrm{V}_{95} \mathrm{~W}_{05}, \mathrm{~V}_{95} \mathrm{Ta}_{05}$, or $\mathrm{V}_{95} \mathrm{Hf}_{05}$ alloys, respectively) plays an important role 
in stabilization of these alloys, a significant increase in $\Delta E_{1}(+0.0086,+0.0348$, and +0.0079 mRy, for the $\mathrm{V}_{95} \mathrm{~W}_{05}, \mathrm{~V}_{95} \mathrm{Ta}_{05}$, or $\mathrm{V}_{95} \mathrm{Hf}_{05}$ alloys, respectively) takes place indicating that increases in $\Delta E_{M}$ and $\Delta E_{1}$ are comparable for the $\mathrm{V}_{95} \mathrm{~W}_{05}$ alloy. However, for the $\mathrm{V}_{95} \mathrm{Ta}_{05}$ alloy the increase in $\Delta E_{1}$ is almost twice as large as that in $\Delta E_{M}$. It appears that for only one alloy that $\mathrm{V}$ forms with $5 d$-transition metals under consideration, $\mathrm{V}_{95} \mathrm{Hf}_{05}$, the electrostatic Madelung energy is the major factor in stabilization of the bcc structure.

According to figure 3 , the band-structure energy is also responsible for the pressureinduced shear softening in pure $\mathrm{Nb}$ at the pressure of $\sim 0.5 \mathrm{Mbar}$ that corresponds to the atomic volume $\Omega=14.92 \AA^{3}$. This pressure is selected to perform calculations for pure niobium and $\mathrm{Nb}$-based alloys. There are obvious analogies when $\mathrm{Nb}$ is alloyed with its $4 d$-neigbors ( $\mathrm{Zr}$ and $\mathrm{Mo}$ ) with the case of $\mathrm{V}$ alloyed with its $3 d$-neigbors ( $\mathrm{Ti}$ and $\mathrm{Cr}$ ): the band-filling argument (the number of valence electrons) is responsible, exclusively, for stabilization or destabilization of the bcc phase when $\mathrm{Nb}$ is alloyed with its neighbors within the same $4 d$-transition series, Mo or $\mathrm{Zr}$, respectively.

In addition to results on the $\mathrm{Nb}_{95} \mathrm{Zr}_{05}$, and $\mathrm{Nb}_{95} \mathrm{Mo}_{05}$ alloys, table I contains the results of calculations of the energy change caused by $1 \%$ of the monoclinic deformation in the alloys that $\mathrm{Nb}$ forms with its $3 d$ neighbors $\left(\mathrm{Nb}_{95} \mathrm{Ti}_{05}, \mathrm{Nb}_{95} \mathrm{~V}_{05}\right.$, and $\left.\mathrm{Nb}_{95} \mathrm{Cr}_{05}\right)$ and $5 d$ neighbors $\left(\mathrm{Nb}_{95} \mathrm{Hf}_{05}\right.$, $\mathrm{Nb}_{95} \mathrm{Ta}_{05}$, and $\mathrm{Nb}_{95} \mathrm{~W}_{05}$ ).

In table III we summarize the calculated charge transfers for $\mathrm{Nb}$ alloyed with the members of Group-IVB, Group-VB, and Group-VIB. As one can see from this table, there is a significant negative charge transfer on the $\mathrm{Nb}$ atoms when $\mathrm{Nb}$ is alloyed with $3 d$ metals $(\mathrm{Ti}, \mathrm{V}$, and $\mathrm{Cr}$ ). The decrease in $\Delta E_{M}$ of pure $\mathrm{Nb}$ due to alloying is proportional to the absolute value of the charge transfer and appears to be $-0.0091,-0.0101$, and $-0.0144 \mathrm{mRy}$ for the $\mathrm{Nb}_{95} \mathrm{Ti}_{05}$, $\mathrm{Nb}_{95} \mathrm{~V}_{05}$, and $\mathrm{Nb}_{95} \mathrm{Cr}_{05}$ alloys, respectively. There is also a decrease in $\Delta E_{1}$ due to alloying: 0.0080, -0.0045, and $-0.0036 \mathrm{mRy}$ for the $\mathrm{Nb}_{95} \mathrm{Ti}_{05}, \mathrm{Nb}_{95} \mathrm{~V}_{05}$, and $\mathrm{Nb}_{95} \mathrm{Cr}_{05}$ alloys, respectively, but in contrast to $\Delta E_{M}$ behavior, the drop in $\Delta E_{1}$ decreases as the absolute value of the charge transfer on the $\mathrm{Nb}$ atoms increases Both the electrostatic Madelung and band-structure contributions determine the decrease in the total-energy, $\Delta E_{\text {tot }}$, of $\mathrm{Nb}$ when it is alloyed with $3 d$ transition metals, but only for the $\mathrm{Nb}_{95} \mathrm{Ti}_{05}$ alloy these contributions are comparable in magnitude and the $\Delta E_{M}$ contribution plays the decisive role in destabilization of the $\mathrm{Nb}_{95} \mathrm{~V}_{05}$, and $\mathrm{Nb}_{95} \mathrm{Cr}_{05}$ alloys.

Table III. The calculated charge transfer induced on the $\mathrm{Nb}$ atoms in Nb-M alloys.

\begin{tabular}{|l|l|l|}
\hline $\mathrm{Nb}-\mathrm{Ti}(-0.146)$ & $\mathrm{Nb}-\mathrm{V}(-0.247)$ & $\mathrm{Nb}-\mathrm{Cr}(-0.336)$ \\
\hline $\mathrm{Nb}-\mathrm{Zr}(0.084)$ & $\mathrm{Nb}-\mathrm{Nb}(0.000)$ & $\mathrm{Nb}-\mathrm{Mo}(-0.081)$ \\
\hline $\mathrm{Nb}-\mathrm{Hf}(0.0 .88)$ & $\mathrm{Nb}-\mathrm{Ta}(0.046)$ & $\mathrm{Nb}-\mathrm{W}(-0.005)$ \\
\hline
\end{tabular}

Due to the similarity between $4 d$ and $5 d$ states, one can explain why an insignificant charge transfer occurs when $4 d$ - $\mathrm{Nb}$ is alloyed with $5 d$-metals $\mathrm{Hf}$, Ta, and W (see table III). Addition of 5 at. $\% \mathrm{Hf}$ or $\mathrm{W}$ decreases the total-energy of pure $\mathrm{Nb}: \Delta E_{\text {tot }}=-0.0155$ and -0.0066 mRy for the $\mathrm{Nb}_{95} \mathrm{Hf}_{05}$ and $\mathrm{Nb}_{95} \mathrm{~W}_{05}$ alloys, respectively, indicating a slight destabilization of the bcc phase. In the case of the $\mathrm{Nb}_{95} \mathrm{Ta}_{05}$ alloy the changes in both $\Delta E_{M}$ and $\Delta E_{1}$ are positive: +0.0020 and $+0.0005 \mathrm{mRy}$, respectively, resulting in a slight $\left(\Delta E_{t o t}=+0.0025 \mathrm{mRy}\right)$ stabilization of the bcc phase in the $\mathrm{Nb}_{95} \mathrm{Ta}_{05}$ alloy. In fact, only Mo and Ta stabilize the bcc structure in $\mathrm{Nb}$ 
but all the other metals under consideration behave differently where the largest degree of destabilization of the bcc phase in $\mathrm{Nb}$ takes place in the $\mathrm{Nb}-\mathrm{Zr}$ system.

The calculations for pure tantalum and Ta-based alloys are performed at the atomic volume $\Omega=14.68 \AA^{3}$ corresponding to the pressure of $\sim 0.65 \mathrm{Mbar}$, which is within the range of the $c_{44}$ softening (figure 3). Again, there are analogies to the case of $\mathrm{V}$ and $\mathrm{Nb}$ when they are alloyed with their nearest neighbors within the same $d$-transition series: the band-filling argument (the number of valence electrons) is responsible, exclusively, for stabilization or destabilization of the bcc phase when Ta is alloyed with its neighbors within the same $5 d$ transition series, $\mathrm{W}$ or $\mathrm{Hf}$, respectively.

In addition to results on the $\mathrm{Ta}_{90} \mathrm{Hf}_{10}$, and $\mathrm{Ta}_{90} \mathrm{~W}_{10}$ alloys, table I contains the results of the present calculations of the energy change caused by $1 \%$ of the monoclinic deformation in the alloys that $\mathrm{Ta}$ forms with its $3 d$ neighbors $\left(\mathrm{Ta}_{90} \mathrm{Ti}_{10}, \mathrm{Ta}_{90} \mathrm{~V}_{10}\right.$, and $\left.\mathrm{Ta}_{90} \mathrm{Cr}_{10}\right)$ and $4 d$ neighbors $\left(\mathrm{Ta}_{90} \mathrm{Zr}_{10}, \mathrm{Ta}_{90} \mathrm{Nb}_{10}\right.$, and $\left.\mathrm{Ta}_{90} \mathrm{Mo}_{10}\right)$. In table IV we summarize the calculated charge transfers for the Ta-based alloys. In analogy with $\mathrm{Nb}$, there is a significant negative charge transfer on the host (Ta) atoms, when Ta is alloyed with $3 d$ metals $(\mathrm{Ti}, \mathrm{V}$, and $\mathrm{Cr}$ ), that provides a decrease in $\Delta E_{M}\left(-0.0056,-0.0105\right.$, and $-0.0133 \mathrm{mRy}$ for the $\mathrm{Ta}_{90} \mathrm{Ti}_{10}, \mathrm{Ta}_{90} \mathrm{~V}_{10}$, and $\mathrm{Ta}_{90} \mathrm{Cr}_{10}$ alloys, respectively), which magnitude is proportional to the magnitude of the charge transfer (see Table IV). A decrease in $\Delta E_{1}$ also takes place $\left(-0.0108,-0.0101\right.$, and $-0.0081 \mathrm{mRy}$ for the $\mathrm{Ta}_{90} \mathrm{Ti}_{10}$, $\mathrm{Ta}_{90} \mathrm{~V}_{10}$, and $\mathrm{Ta}_{90} \mathrm{Cr}_{10}$ alloys, respectively), but, again as it was in the case of $\mathrm{Nb}-3 d$ metals based alloys, opposite to $\Delta E_{M}$ behavior: the drop in $\Delta E_{l}$ decreases as the absolute value of the charge transfer on the Ta atoms increases. Consequently, there is a significant decrease (softening) in the total-energy change when Ta is alloyed with $3 d$ metals $(-0.0164,-0.0206$, and $-0.0214 \mathrm{mRy}$ for the $\mathrm{Ta}_{90} \mathrm{Ti}_{10}, \mathrm{Ta}_{90} \mathrm{~V}_{10}$, and $\mathrm{Ta}_{90} \mathrm{Cr}_{10}$ alloys, respectively). The largest decrease ( 9.9\%) occurs in the $\mathrm{Ta}_{90} \mathrm{Cr}_{10}$ alloy corresponding to the greatest negative charge transfer on the Ta atoms.

Table IV. The calculated charge transfer induced on the Ta atoms in Ta-M alloys.

\begin{tabular}{|l|l|l|}
\hline Ta-Ti (-0.193) & Ta-V (-0.296) & Ta-Cr (-0.386) \\
\hline Ta-Zr (0.041) & Ta-Nb (-0.046) & Ta-Mo (-0.127) \\
\hline Ta-Hf (0.0.44) & Ta-Ta (0.000) & Ta-W (-0.051) \\
\hline
\end{tabular}

Finally, when Ta is alloyed with $4 d$-metals ( $\mathrm{Zr}$ and $\mathrm{Mo}$ ) the largest drop in $\Delta E_{M}$ due to alloying (-0.0071 $\mathrm{mRy})$ is observed in the $\mathrm{Ta}_{90} \mathrm{Mo}_{10}$ alloy where a relatively large negative charge transfer on the Ta atoms (-0.127) takes place. For the $\mathrm{Ta}_{90} \mathrm{Zr}_{10}$ alloy, where the charge transfer on the Ta atoms is positive $(+0.041)$, the change in $\Delta E_{M}$ is positive $(+0.0027 \mathrm{mRy})$. A decrease in $\Delta E_{1}$ also occurs $\left(-0.0143\right.$ and $-0.0050 \mathrm{mRy}$ for the $\mathrm{Ta}_{90} \mathrm{Zr}_{10}$ and $\mathrm{Ta}_{90} \mathrm{Mo}_{10}$ alloys, respectively). This situation is similar to that when Ta is alloyed with $3 d$-metals: the drop in $\Delta E_{l}$ decreases as the absolute value of the charge transfer on the Ta atoms increases. The decrease in both $\Delta E_{1}$ and $\Delta E_{M}$ in the $\mathrm{Ta}_{90} \mathrm{Mo}_{10}$ alloy and a significant decrease in $\Delta E_{1}$, which overcomes a slight increase in $\Delta E_{M}$ in the $\mathrm{Ta}_{90} \mathrm{Zr}_{10}$ alloy, result in a decrease in the total-energy in the $\mathrm{Ta}_{90} \mathrm{Mo}_{10}$ and $\mathrm{Ta}_{90} \mathrm{Zr}_{10}$ alloys (-0.0121 and $-0.0116 \mathrm{mRy}$, respectively).

Alloying Ta with 10 at. $\%$ of $\mathrm{Nb}$ increases $\Delta E_{M}(+0.0066 \mathrm{mRy})$ and decreases $\Delta E_{1}$ $(-0.0023 \mathrm{mRy})$ resulting in increase of the total-energy, $\Delta E_{t o t},(+0.0043 \mathrm{mRy})$. The increase in $\Delta E_{M}$ occurs in spite of the small but negative charge transfer on the Ta atoms $(-0.046)$. These values are numerically very small and represent an exception to the rule of thumb we have 
discovered: the sign (positive or negative) of the change of the $\Delta E_{M}$ due to alloying of two transition metals from the different $d$-series corresponds to the sign (positive or negative) of the charge transfer on the host atoms. As a result, $\mathrm{Nb}$ joins $\mathrm{W}$ as the only metals under consideration that stabilize the bcc structure of Ta.

\section{DISCUSSION}

We found that small amounts ( 3-5 at. \%) of $4 d$ and $5 d$ metals are enough to stabilize the bcc structure of V. For these alloys the Madelung energy plays an important role in the stabilization, as opposed to the $\mathrm{V}-\mathrm{Ti}$ and $\mathrm{V}-\mathrm{Cr}$ alloys where it is negligible. This contrasting behavior is due to the inherent difference between $4 d$ and $5 d$ states compared to the $3 d$ states. The former are more extended in space as are their corresponding charge distribution.

Consequently, there is a larger charge transfer when $\mathrm{V}$ is alloyed with a $4 d$ or $5 d$ metal than with its neighbors $\mathrm{Ti}$ and $\mathrm{Cr}$. As can be seen from Table II, the large and positive charge transfers means that more charge is supplied into the interstitial region thereby increasing the Madelung energy of the crystal that favors higher symmetry structures. Let us note that an addition of $4 d$ or $5 d$-metal dopants to $\mathrm{V}$ also affects the band-structure energy, due to the fact that they provide stronger hybridization but, as one can see from table I for the case of $\mathrm{V}$ alloyed by $\mathrm{Zr}, \mathrm{Nb}$, and $\mathrm{Hf}$, this effect is not as important as the change in the Madelung energy, but becomes compatible with the latter for the case of $\mathrm{V}$ alloyed with $\mathrm{W}$ and Mo, and even plays the dominant factor for the bcc structure stabilization in the V-Ta alloys.

The Madelung energy plays an opposite role (destabilization of the bcc phase) when $4 d$ $\mathrm{Nb}$ or $5 d$-Ta metals are alloyed with $3 d$ metals ( $\mathrm{Ti}, \mathrm{V}$, and $\mathrm{Cr}$ ). Because of the above mentioned inherent difference between $4 d$ and $5 d$ states compared to the $3 d$ states, there is a significant negative charge transfer on the $\mathrm{Nb}$ (Ta) atoms (see Tables III and IV) when $\mathrm{Nb}(\mathrm{Ta}$ ) is alloyed with $3 d$ metals ( $\mathrm{Ti}, \mathrm{V}$, and $\mathrm{Cr}$ ). The large and negative charge transfers means that less charge is supplied into the interstitial region thereby decreasing the electrostatic Madelung energy contribution that favors destabilization of the high symmetry structure (the bcc phase). An addition of $3 d$-metal dopants to $\mathrm{Nb}(\mathrm{Ta})$ also affects the band structure energy, due to the fact that they provide stronger hybridization, also contributing to destabilization of the bcc phase. Nevertheless, in spite of some negative contribution (due to alloying with $3 d$ metals) to both $\Delta E_{1}$ and $\Delta E_{M}$, the $\mathrm{Nb}$ - and Ta-based alloys remain stable in the bcc structure due to the enough large value of $\Delta E_{\text {tot }}$ in pure metals (especially in Ta).

Niobium and tungsten are the only metals under consideration that stabilize the bcc structure of Ta. Both Ta-Nb and Ta-W alloys have unlimited range of the bcc solid solutions [27, 28]. It is well known that impurities and alloying can have a profound influence on the yield strength properties of a material [29]. Schwartz et al. [30] showed that adding $10 \mathrm{wt}$. \% (9.86 at. $\%$ ) of $\mathrm{W}$ to Ta increases the yield strength of the materials, i.e. the minimum stress that is required to induce the plastic deformation, by more than a factor of two. This observation was confirmed by Yang et al. [29] who calculated the elastic constants of the $\mathrm{Ta}_{100-\mathrm{x}} \mathrm{W}_{\mathrm{x}}$ alloys and successfully reproduced the experimental concentration behavior of the trigonal shear elastic constant, $c_{44}$, [31]. Our calculations also confirm a gradual increase of the trigonal shear elastic constant, $c_{44}$, of the $\mathrm{Ta}_{100-\mathrm{x}} \mathrm{W}_{\mathrm{x}}$ alloys together with $\mathrm{W}$ content observed in Ref. [31]. We also found a similar behavior in the $\mathrm{Ta}_{100-\mathrm{x}} \mathrm{Nb}_{\mathrm{x}}$ alloys as the amount of $\mathrm{Nb}$ in the alloy increases, but 
this result requires experimental confirmation. Based on our results, we recommend alloying Ta with $\mathrm{Nb}$ for strengthening.

\section{CONCLUSIONS}

To summarize, we have identified that the band-structure structure peculiarities are responsible for the Kohn anomaly on the transverse acoustic phonon mode TA [ $\xi 00$ ] of the Group-VB transition metals and softening in their shear elastic constant with pressure. As far as reliable data on the phonon dispersions for $\mathrm{V}$ became recently available [4], it is now become possible to verify predictions made in Refs. [7,8] that the nesting vector spanning two flat pieces of the FS of vanadium already exists at ambient pressure and leads to the Kohn anomaly on the transverse acoustic phonon mode TA [ $\xi 00]$. In the present study we prove that the length of the above mentioned nesting vector matches the position where the Kohn anomaly (the maximum deviation from the elastic constant line) on the experimental TA [ $\xi 00]$ phonon branch occurs. Our calculations confirm that this anomaly takes place at $\xi \approx 0.24,0.16$, and 0.28 for $\mathrm{V}, \mathrm{Nb}$, and Ta, respectively that is in a good accord with experimental observations.

For $\mathrm{V}$ this softening is associated with a phase transition to a rhombohedral phase close to $60 \mathrm{GPa}$ while for $\mathrm{Nb}$ and $\mathrm{Ta}$ the bcc phase still remains stable at all studied pressures. The effect of alloying on the phase stability has been studied and three dominant mechanisms are recognized. First, the band-structure energy tends to destabilize or stabilize the bcc phase when a member of the Group-VB is alloyed with its nearest neighbor from the same $d$-transition series to left or to the right, respectively. Second, the increase in both the electrostatic Madelung and band-structure energies remove softening and secure the bcc phase when vanadium metal is alloyed with neighboring elements from a higher $d$-transition series. Third, the decrease in both the electrostatic Madelung and band-structure energies causes some destabilization of the bcc phase when niobium or tantalum metals are alloyed with neighboring elements from the $3 d$ transition series although this destabilization is too small to cause a phase transition. Finally, a slight destabilization of the bcc phase occurs when niobium metal is alloyed with $\mathrm{Hf}$ or $\mathrm{W}$ or when tantalum metal is alloyed with $\mathrm{Zr}$ and Mo but both $\mathrm{Nb}$ and Ta remain stable in the bcc structure. The stability of the bcc phase is only strengthened when the 'mixed' ( $4 d$ and $5 d$ ) alloy has components from the same Group-VB (the Nb-Ta system).

\section{ACKNOWLEDGMENTS}

Work performed under the auspices of the U.S. DOE by LLNL under contract DE-AC5207NA27344.

\section{REFERENCES}

1. M. Ishizuka, M. Iketani, and S. Endo, Phys. Rev. B 61, R3823 (2000).

2. N. Suzuki and M. Otani, J. Phys.: Condens. Matter 14, 10869 (2002). 
3. W. Luo, R. Ahuja, Y. Ding, and H. K. Mao, Proc. Natl. Acad. Sci. 104, 16428 (2007).

4. A. Bosak, M. Hoesch, D. Antonangeli, D. L. Farber, I. Fischer, and M. Krisch, Phys. Rev. B 78, 020301(R) (2008).

5. Y. Ding, R. Ahuja, J. Shu, P. Chow, W. Luo, and H. K. Mao, Phys. Rev. Lett. 98, 085502 (2007).

6. B. Lee, R. E. Rudd, J. E. Klepeis, P. Söderlind, and A. Landa, Phys. Rev. B 75, (2007) 180101(R).

7. A. Landa, J. Klepeis, P. Söderlind, I. Naumov, O. Velikokhatnyi, L. Vitos, and A. Ruban, J. Phys.: Condens. Matter 18, 5079 (2006).

8. A. Landa, J. Klepeis, P. Söderlind, I. Naumov, O. Velikokhatnyi, L. Vitos, and A. Ruban, J. Phys. Chem. Sol. 67, 2056 (2006).

9. L. Koči, Y. Ma, A. R. Oganov, P. Souvatzis, and R. Ahuja, Phys. Rev. B 77, 214101 (2008).

10. V. V. Struzhkin, Y. A. Timofeev, R. J. Hemley, and H. K. Mao, Phys. Rev. Lett. 79, 4262 (1997).

11. B. T. Matthias, T. H. Geballe, and V. B. Compton, Rev. Mod. Phys. 35(1), 1 (1963).

12. Y. Nakagawa and A. D. B. Woods, Phys. Rev. Lett. 11, 271 (1963).

13. A. D. B. Woods, Phys. Rev. 136, A781 (1964).

14. L. Vitos, Computational Quantum Mechanics for Materials Engineers: The EMTO Method and Applications (Springer-Verlag, London, 2007).

15. J. P. Perdew, K. Burke, and M. Ernzerhof, Phys. Rev. Lett. 77, 3865 (1996).

16. J. S. Faulkner, Prog. Mat. Sci. 27, 1 (1982).

17. L. Vitos, I. A. Abrikosov, B. Johansson, Phys. Rev. Lett. 87, 156401 (2001).

18. D. J. Chadi and M. L. Cohen, Phys. Rev B 8, 5747 (1973); S. Froyen, ibid. 39, 3168 (1989).

19. A. V. Ruban and H. L. Skriver, Phys. Rev. B 66, 024201 (2002); A. V. Ruban, S. I. Simak, P. A. Korzhavyi, and H. L. Skriver, ibid. 66, 024202 (2002).

20. I. A. Abrikosov, S. I. Simak, B. Johansson, A. V. Ruban, and H. L. Skriver, Phys. Rev. B 56, 9319 (1997).

21. J. M. Wills, O. Eriksson, M. Alouani, D. L. Price, in: Electronic Structure and Physical Properties of Solids: The Uses of the LMTO Method, edited by H. Dreyssé (SpringerVerlag, Berlin, 2000), pp.148-167.

22. F. D. Murnaghan, Proc. Natl. Acad. Sci. 30, 244 (1944).

23. M. Mehl, B. M. Klein, and D. A. Papaconstantopoulos, in: Intermetallic Compounds: Principles and Practice, Principles, edited by J.H. Westbrook and R.L. Fleisher (Wiley, London, 1995), Vol. 1, pp. 195-210.

24. J. Rath and A. J. Freeman, Phys. Rev. B 11, 2109 (1975).

25. A. Bosak, private communication, (2010).

26. B. M. Powell, R. Martel, and A. D. B. Woods, Phys. Rev. 171, 727 (1968); Can. J. Phys. 55, 1601 (1977).

27. R. Krishnan, S. P. Garg, and N. Krishamurthy, in: Binary Alloy Phase Diagrams, $2^{\text {nd }}$ ed., edited by T. B. Massalski (ASM International, Materials Park, OH, 1990), Vol. 3, p. 2772.

28. R. Krishnan, S. P. Garg, and N. Krishamurthy, in: Binary Alloy Phase Diagrams, $2^{\text {nd }}$ ed., edited by T. B. Massalski (ASM International, Materials Park, OH, 1990), Vol. 3, p. 3438.

29. L. Yang, H. Cynn, J.-H. Klepeis, J. Pask, and R. Rudd, in: Abstracts of APS March Meeting, (Portland, OR, 2010), Bull. Am. Phys. Soc. 55(2), p. 389 (2010).

30. A. J. Schwartz, D. H. Lassila, and M. M. LeBlanc, Mater. Sci. and Eng. A 244, 178 (1998).

31. C. E. Anderson and F. R. Brotzen, J. Appl. Phys. 53(1), 292 (1982). 